\title{
Neurosteroid Synthesis in Adult Female Rat Hippocampus, Including Androgens and Allopregnanolone
}

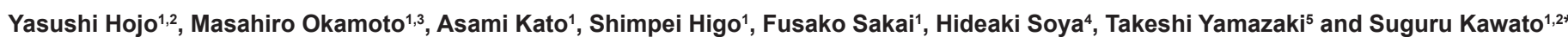

${ }^{1}$ Department of Biophysics and Life Sciences, Graduate School of Arts and Sciences, The University of Tokyo, 3-8-1 Komaba, Meguro, Tokyo 153-8902, Japan, 9 The University of Tokyo, Japan

${ }^{2}$ Bioinformatics Project of Japan Science and Technology Agency, The University of Tokyo, Japan

${ }^{3}$ Research Fellow of the Japan Society for the Promotion of Science, Japan

${ }^{4}$ Laboratory of Exercise Biochemistry and Neuroendocrinology, Institute for Health \& Sports Sciences, University of Tsukuba, Tsukuba, Ibaraki, Japan

${ }^{5}$ Laboratory of Molecular Brain Science, Graduate School of Integrated Arts and Sciences, Hiroshima University, Higashi-Hiroshima, Japan

\begin{abstract}
Female rat hippocampus synthesizes significant amount of estrogens, including progesterone (PROG), estrone and estradiol (E2). Hippocampal level of PROG and E2 are considerably higher than those in plasma. Female hippocampal estrogens play a significant role in the fluctuation of dendritic spine density across the estrous cycle. Here we extend the study to the investigation of female androgens, including testosterone (T) and Dihydrotestosterone (DHT), in the female rat hippocampus, since female androgens had been largely unknown. By combination of mass-spectrometric analysis with HPLC-purification and picolinoyl-derivatization of sex steroids, we determined the accurate concentration of $\mathrm{T}$ and $\mathrm{DHT}$ in the hippocampus. The levels of $\mathrm{T}$ and $\mathrm{DHT}$ in female hippocampus at Proestrus were approximately $1.1 \mathrm{nM}$ and $0.6 \mathrm{nM}$, respectively, suggesting a significant synthesis of T and DHT. The level of plasma T was approx. $0.1 \mathrm{nM}$, implying almost no contribution of plasma T to hippocampal T. The concentration of hippocampal DHT had a good correlation with that of hippocampal T, suggesting a significant activity of $5 \alpha$-reductase (DHT synthase) in the female. Allopregnanolone level was also determined as a useful indicator of 5a-reductase activity. Interestingly mRNA expression level of $5 \alpha$-reductase and androgen receptor $(A R)$ was not significantly different between the different estrous cycle stages, or between female and male. Nevertheless, sex difference existed with respect to the levels of $\mathrm{T}, \mathrm{DHT}$ and Allo in hippocampus. Although physiological significance of female hippocampal androgens waits further investigations, the female hippocampus produces T or DHT which may be useful to suppress anxiety, for example.
\end{abstract}

Keywords: Dihydrotestosterone; Female hippocampus; Androgens; Testosterone; Allopregnanolone

Abbreviations: Allo: Allopregnanolone; AR: Androgen Receptor; D1: Diestrus 1; D2: Diestrus 2; DHT: Di Hydro Testosterone; E2: Estradiol; ER: Estrogen Receptor; Est: Estrus; LC-MS/MS: Liquid Chromatography with Tandem-Mass-Spectrometry; Pro: Proestrus; PROG: Progesterone; PFBz: Pentafluorobenzoxy; StAR: Steroidogenic Acute Regulatory Protein; T: Testosteron

\section{Introduction}

In female hippocampus, synthesis and function of androgens have not been well investigated, although estrogens have been extensively studied about synthesis and function. We recently demonstrated that female rat hippocampus synthesizes $17 \beta$-estradiol (E2), progesterone (PROG) and estrone [1], which is similar to hippocampus-synthesized steroids in male [2-5]. The estrous cycle-dependent fluctuation of the spine density in female rat hippocampus had a good correlation with the cyclic fluctuation of hippocampal E2 or PROG level [1]. Hippocampal testosterone (T) level in female rats was almost as high as hippocampal E2 level ( 1 nM) [1]. The level of Dihydrotestosterone (DHT), the most potent androgen, in female hippocampus, however, remains to be undetermined, although one study reported the presence of very low level DHT (the numerical concentrations were not written) with radioimmunoassay (RIA) [6]. We should determine the accurate concentration of DHT by improvement of the sensitivity of measurements in female hippocampus, since androgen application to the aged female hippocampus decrease a depression-like behavior of aged mice [7]. Concerning the steroid synthesis systems in female rat hippocampus, mRNA for $\mathrm{T}$ synthesis including $\mathrm{P} 450$ (17 $\alpha)$ and $17 \beta$-HSD (type 1 and 3 ) are expressed [1]. It remains unclear whether $5 a$-reductase (DHT synthase) and androgen receptor (AR) are expressed in female hippocampus, although one report showed $5 \alpha$-reductase (type 2) expression with in situ hybridization in female mice brain [8]. In male rat hippocampus, steroidogenic enzymes and steroid receptors are mainly localized in pyramidal neurons in CA1 and CA3, and granule neurons in dentate gyrus [2,4,9-12]. Their neuronal localization of female should be clarified.

$5 a$-reductase produces not only DHT from $\mathrm{T}$, but also allopregnanolone [13] from PROG. Concentration of Allo has been demonstrated using RIA [6,14], GC-MS [15,16] and liquid chromatography with tandem-mass-spectrometry (LC-MS/MS) [17]. Although Allo had been extensively investigated with regard to its anxiolytic and anti-depressive effects $[18,19]$ via modulation of gamma-aminobutyric acid A (GABAA) receptors [20], the physiological significance of DHT in female hippocampus is still unknown and should be investigated. We here examine whether DHT synthase or AR expresses in female rat hippocampus. Localization of P450 (17a), steroidogenic acute regulatory protein (StAR) and AR in female hippocampus is also examined with Immunohistochemical

*Corresponding author: Suguru Kawato, Department of Biophysics and Life Sciences, Graduate School of Arts and Sciences, The University of Tokyo, 3-81 Komaba, Meguro, Tokyo 153-8902, Japan, Tel/Fax: 81-3-5454-6517; E-mail: kawato@bio.c.u-tokyo.ac.jp

Received December 01, 2013; Accepted December 30, 2013; Published January 10,2014

Citation: Hojo Y, Okamoto M, Kato A, Higo S, Sakai F, et al. (2014) Neurosteroid Synthesis in Adult Female Rat Hippocampus, Including Androgens and Allopregnanolone. J Steroids Hormon Sci S4: 002. doi:10.4172/2157-7536.S4-002

Copyright: (c) 2014 Hojo Y, et al. This is an open-access article distributed under the terms of the Creative Commons Attribution License, which permits unrestricted use, distribution, and reproduction in any medium, provided the original author and source are credited. 
staining. By combination of mass-spectrometric analysis with HPLCpurification and picolinoyl-derivatization of steroids [1,10,21], the accurate concentration of hippocampal DHT and T is determined. Allo in female hippocampus is determined to compare with male Allo [6,1417]. By comparison of hippocampal androgens and Allo between in male and female, we clarified the profile of steroid synthesis in female rat hippocampus, which is different from male.

\section{Materials and Methods}

\section{Animals}

Wistar rats (10 weeks old) were purchased from Saitama Experimental Animals Supply (Japan). The estrous cycle of female rats was monitored with morning vaginal smears. Only those rats showing three consecutive 4-day cycles of Pro, Est, D1 and D2 were used at the age of 12 weeks old. Ovariectomy (OVX) and sham operations were performed two-weeks before (at 10w old) the experiments. Male rats were also used at the age of 12 weeks old. All animals were maintained under a $12 \mathrm{~h}$ light $/ 12 \mathrm{~h}$ dark exposure and free access to food and water. The experimental procedure of this research was approved by the Committee for Animal Research of the University of Tokyo.

\section{Chemicals}

$\mathrm{T}$ and DHT were purchased from Sigma [22]. Picolinic acid was from Tokyo Chemical Industry (Japan) and, ${ }^{13} \mathrm{C}_{3}-\mathrm{T},{ }^{13} \mathrm{C}_{3}$-DHT and Allo- $\mathrm{d}_{4}$ were from $\mathrm{CDN}$ Isotope Inc. (Canada). $\left[{ }^{3} \mathrm{H}\right]$ labeled steroids $\left(\left[1,2,6,7-{ }^{3} \mathrm{H}\right]-\mathrm{T}, \quad\left[1,2,6,7-{ }^{3} \mathrm{H}\right]-\mathrm{DHT}\right.$ and $\left.\left[1,2,6,7-{ }^{3} \mathrm{H}\right]-\mathrm{Allo}\right)$ were purchased from Perkin Elmer [22].

\section{RT-PCR}

The detailed procedures of mRNA analyses are described in elsewhere $[2,4,23]$. Total RNAs were isolated from the hippocampus adrenal gland, liver, prostate and testis of adult rats, using a SV total RNA Isolation System (Promega, USA). The purified RNAs were treated with RNase-free DNase to eliminate the possibility of genomic DNA contamination, and quantified on the basis of the absorbance at 260/280 $\mathrm{nm}$. The purified RNAs (100 ng) were reverse-transcribed to obtain cDNAs, using a M-MLV Reverse Transcriptase (Promega, USA). PCR was performed by using these cDNAs. The oligonucleotides for PCR amplification were designed as illustrated in Table S1. The PCR protocols comprised application of a $30 \mathrm{~s}$ denaturation period at $95^{\circ} \mathrm{C}$, a $20 \mathrm{~s}$ annealing period at individual temperature for each enzyme, and a $30 \mathrm{~s}$ extension at $72^{\circ} \mathrm{C}$, for individual number of cycles for each enzyme (Table S1). For semi-quantitative analysis, the RT-PCR products were separated on $2 \%$ agarose gels, stained with ethidium bromide, and analyzed with a fluorescence gel scanner (Atto, Japan) and Image J software. In all the cases we first plotted amplification curves in order to choose the linear phase of PCR cycles. The comparison between different estrous cycle stages can be performed after normalization by glyceraldehyde-3-phosphate dehydrogenase (GAPDH, a house keeping gene) as internal standard. It should be noted that GAPDH expression was not changed across the estrous cycle. The optimal cycle number of GAPDH mRNA was determined as 17 from the amplification curve. The comparison of relative abundance between different estrous stages or between female and male was performed by using the normalized expression. As positive control, we used the reference organ (adrenal gland, liver, prostate and testis)

\section{Mass-spectrometric assay of steroids (T, DHT and Allo)}

Detailed procedures are described elsewhere [21].
Step 1: Purification of steroids from hippocampi with normal phase HPLC.

A rat was deeply anesthetized and decapitated at 10-10:30 a.m., since at this time window estradiol (E2) surge at Pro occurs in plasma [24]. The whole hippocampi was removed and homogenized. To calculate the recovery of steroids, radioactive steroids $(20,000 \mathrm{cpm})$ were added as internal standards to hippocampal homogenate. To extract steroid metabolites, ethyl acetate/hexane $(3: 2 \mathrm{vol} / \mathrm{vol})$ was applied to the homogenates which were then mixed. The mixture was centrifuged at 2,500×g and the organic layer was collected. After evaporation, the extracts were dissolved in $1 \mathrm{ml}$ of $40 \%$ methanol $/ \mathrm{H}_{2} \mathrm{O}$ and applied to a $\mathrm{C}_{18}$ Amprep solid phase column (Amersham Biosciences, USA) to remove contaminating fats. The extracts were dried, dissolved in an elution solvent of HPLC. The steroid metabolites were separated into T and DHT using a normal phase HPLC system (Jasco, Japan) with an elution solvent of hexane: isopropyl alcohol: acetic acid=98:2:1. A silica gel column $(0.46 \times 15 \mathrm{~cm}$, Cosmosil 5SL, Nacalai Tesque, Japan) was used. By monitoring $3 \mathrm{H}$-steroids, the recoveries of T, DHT and Allo were $41 \pm 7 \%, 29 \pm 5 \%$ and $50 \pm 8 \%$ respectively, after extraction, $\mathrm{C}_{18}$ column treatment and normal phase HPLC separation. As internal standards, $100 \mathrm{pg}$ of isotope labeled steroids $\left({ }^{13} \mathrm{C}_{3}-\mathrm{T},{ }^{13} \mathrm{C}_{3}\right.$-DHT and Allo- $\mathrm{d}_{4}$ ) were added to steroid extracts.

Step 2: Derivatization of HPLC-purified steroids before application to LC (reverse 185 phase)-MS/MS.

Preparation and purification of T-17-picolinoyl-ester, DHT-17picolinoyl-ester and Allo-3-picolinoyl-ester were performed with slight modification of previous methods [1,21]. For preparation of T-17picolinoyl-ester, DHT-17-picolinoyl-ester and Allo-3-picolinoyl-ester, evaporated steroid extracts from the hippocampus or plasma were reacted with $50 \mu \mathrm{L}$ of picolinoic acid suspension (4\% 191 picolinoic acid, $4 \%$ of 4-dimethylaminopyridine, 2\% 2-methyl-6-nitrobenzoic anhydride in tetrahydrofuran anhydrous) (i.e., $80 \mathrm{mg}$ of picolinoic acid, $80 \mathrm{mg}$ of 4-dimethylaminopyridine, $40 \mathrm{mg}$ of 2-methyl-6-nitrobenzoic acid in $2 \mathrm{~mL}$ of tetrahydrofuran) and $20 \mu \mathrm{L}$ of triethylamine, for 0.5 $\mathrm{h}$ at room temperature. The reaction products dissolved in $1 \%$ acetic acid were purified using a Bond Elute $\mathrm{C}_{18}$ column (Varian, USA). The dried sample was dissolved in elution solvent of LC. The reaction products were purified with the $C_{18}$ column by using $80 \%$ acetonitrile. The purified steroid-derivative was dissolved in elution solvent of LC.

Step 3: Determination of the concentration for T, DHT and Allo using LC-MS/MS.

For determination of the concentration of T and DHT, the LC-MS/ MS system, which consists of a Shimadzu HPLC system and an API5000 triple stage quadrupole mass spectrometer (Applied Biosystems, USA) were employed. LC chromatographic separation was performed on a Kinetex $\mathrm{C}_{18}$ column $(2.1 \times 150 \mathrm{~mm}, 1.7 \mu \mathrm{m}$, Phenomenex, USA) for T-picolinoyl ester and DHT-picolinoyl ester. For determination of the concentration of Allo, LC chromatographic separation was performed on a preparation column (Unison UK- Phenyl HT ( $3 \mu \mathrm{m}, 2.0 \times 50 \mathrm{~mm}$, Imtact, Japan)) and an analytical column (Cap cell core $\mathrm{C}_{18}(2.7 \mu \mathrm{m}$, $2.1 \times 100 \mathrm{~mm}$, Shiseido Japan)). After elution of Allo-picolinoyl-ester from the preparation column, Allo-picolinoyl-ester was subsequently loaded to the analytical column. The preparation column enables us to separate Allo (3a-hydroxy-5a-pregnan-20-one) from isomers with equivalent molecular weight ( $3 \alpha$-hydroxy- $5 \beta$-pregnan-20-one, $3 b$-hydroxy-5a-pregnan-20-one and 3b-hydroxy-5 $\beta$-pregnan-20one). MS analysis was operated with electro spray ionization [25] in the positive-ion mode. The isotope-labeled steroid derivatives were 
Citation: Hojo Y, Okamoto M, Kato A, Higo S, Sakai F, et al. (2014) Neurosteroid Synthesis in Adult Female Rat Hippocampus, Including Androgens and Allopregnanolone. J Steroids Hormon Sci S4: 002. doi:10.4172/2157-7536.S4-002

used for calibration of retention time by monitoring the $\mathrm{m} / \mathrm{z}$ transition, from 397 to 256 for ${ }^{13} \mathrm{C}_{3}$-T-picolinoyl-ester, from 399 to 206 for ${ }^{13} \mathrm{C}_{3}$ DHT-picolinoyl-ester, and from 428.4 to 287.3 for Allo-d4-picolinoylester, respectively. Isotope-labeled steroid derivatives were used for internal standards in order to measure recovery of steroids as well as to calibrate the retention time. By monitoring isotope steroids, the recoveries of T, DHT and Allo were determined as $73 \pm 3 \%, 70 \pm 2 \%$ and $82 \pm 6 \%$, respectively, after derivatization, purification and MS/MS detection. In the multiple reaction monitoring mode, the instrument monitored the $\mathrm{m} / \mathrm{z}$ transition, from 394 to 253 for T-picolinoyl, from 396 to 203 for DHT-picolinoyl, and from 424.4 to 283.3 for Allo-picolinoyl, respectively (Figure S1 and Table S2). Here, $\mathrm{m}$ and $\mathrm{z}$ represent the mass and charge of a steroid derivative, respectively. The limits of quantification for steroids were measured with blank samples, prepared alongside hippocampal samples through the whole extraction, fractionation and purification procedures. The limits of quantification were $1 \mathrm{pg}$ for T and DHT, and $0.5 \mathrm{pg}$ for Allo per $0.1 \mathrm{~g}$ of hippocampal tissue, respectively (Table S2). From the calibration curve using standard T or DHT dissolved in blank samples, the linearity was observed between $1.0 \mathrm{pg}$ and $2000 \mathrm{pg}$ for T and DHT, between $5 \mathrm{pg}$ and $1000 \mathrm{pg}$ for Allo, respectively (Figure S2).

\section{Immunohistochemical staining of hippocampal slices}

Detailed procedures are described elsewhere [2,4,10,11]. Hippocampal slices were prepared from 12 week-old female rats at Proestrus stage. Animals were deeply anesthetized and perfused transcardially with PBS (0.1 M phosphate buffer and $0.14 \mathrm{M} \mathrm{NaCl}, \mathrm{pH}$ 7.3), followed by fixative solution ( $4 \%$ paraformaldehyde/PBS). The hippocampi were post fixed, cryo protected, and frozen-sliced coronally with a cryostat (CM1510, Leica). Staining for steroidogenic enzymes (P450 (17a), P450arom and StAR) and receptors (AR and ERa) was performed with the avidin-biotin-peroxidase complex technique. Dilution of primary antibody was 1:1000 each for purified anti-guinea pig cytochrome P450(17a) IgG [26], purified anti-human P450arom IgG [27], anti-mouse StAR IgG [28], anti-AR IgG (PG-21, Millipore, USA), or purified anti-rat C terminal of ERa antibody (RC-19) [12]. After application of primary antibody, the slices were incubated for 18 $\mathrm{h}$ at $4^{\circ} \mathrm{C}$, in the presence of $0.5 \%$ Triton X-100. Biotinylated anti-rabbit IgG (1:1000) and streptavidin-horseradish peroxidase complex (Vector Laboratories) was applied. Immuno-reactive cells were detected with a solution of diaminobenzidine with ammonium nickel sulfate. After embedding in Entellan Neu (Merck, Germany), the immuno-reactive cells were observed under microscope.

\section{Statistical analysis}

Data are expressed as mean \pm SEM. For comparison of the mRNA level of steroidogenic enzymes and receptors, we used a 1-way ANOVA followed by Tukey-Kramer posthoc multiple comparisons. For comparison of the concentration of T or DHT in the hippocampus between male and female, we used Student's t-test. A difference was considered significant at a value of ${ }^{*} p<0.05,{ }^{* *} p<0.01$ or ${ }^{* * *} p<0.001$.

\section{Results}

\section{Molecular biological analysis of steroidogenic enzymes and steroid receptors in the female hippocampus}

The hippocampal expression of steroidogenic enzymes (StAR, $5 a$-reductase (Types 1 and 2)) and AR were examined across the estrous
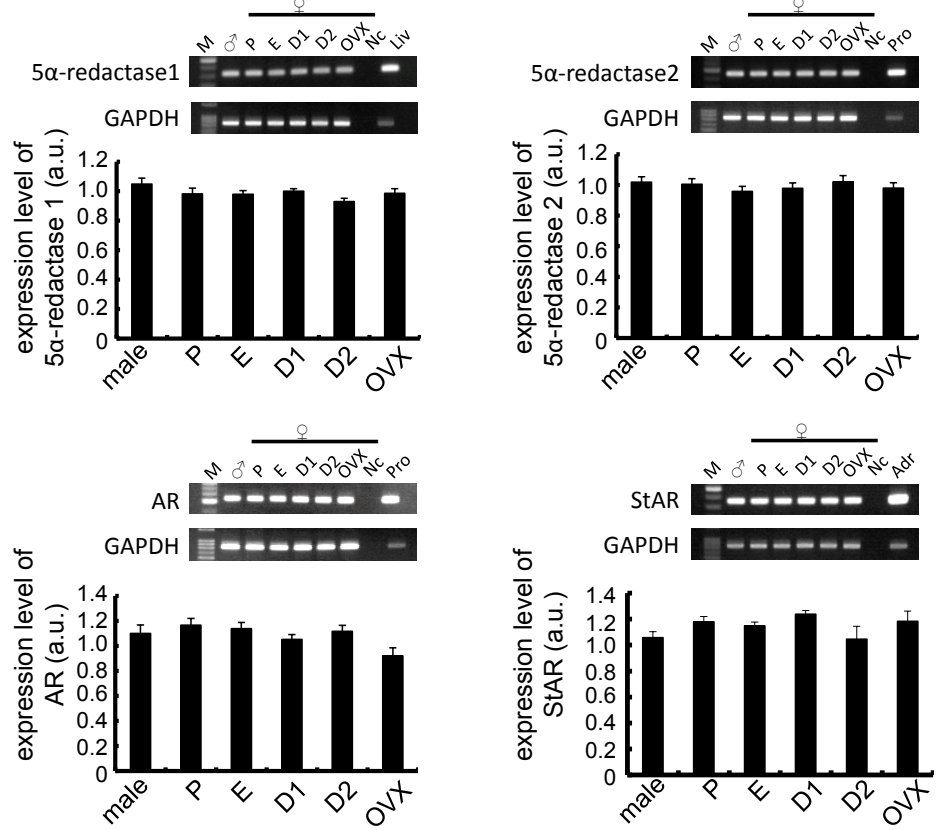

Figure 1: No significant change in expression levels of mRNAs for sex steroidogenic enzymes and receptor (A: 5a-reductase type1, B: $5 a-$ reductase type2, C: AR and D: StAR) in the female hippocampus across the estrous cycle. Upper panels show representative PCR images and lower panels show statistical comparisons. In each images, from left to right, size marker (100 bp ladder) (M), male hippocampus (male), female hippocampus at Pro (P), Est (E), D1 (D1), D2 (D2), and OVX female rats (OVX), the sample without template DNA as negative control (Nc). $F$ o $r$ each enzyme, the RT-PCR products for mRNAs are visualized with ethidium bromide staining on the top of each panel. As an internal control, the ethidium bromide staining of GAPDH is shown on the bottom of each panel. PCR was performed by using cDNA made by reverse transcription from $100 \mathrm{ng}$ of hippocampal total RNA. Statistical comparisons show no estrous cycle-dependent changes of mRNA expression for sex-steroidogenic enzymes. The vertical axis indicates the expression level for each enzyme calculated from the intensity of EB bands. Each value is mean \pm S.E.M. Data are taken from duplicate determinations for each rat of total 4 rats. 
Citation: Hojo Y, Okamoto M, Kato A, Higo S, Sakai F, et al. (2014) Neurosteroid Synthesis in Adult Female Rat Hippocampus, Including Androgens and Allopregnanolone. J Steroids Hormon Sci S4: 002. doi:10.4172/2157-7536.S4-002

cycle. Expression levels of mRNA transcripts normalized by GAPDH are shown as bar graphs in Figure 1. Surprisingly, no significant cyclic fluctuations across the estrous cycle were observed for their expression levels. OVX did not change the expression level of any steroidogenic enzyme and AR. Relative number of transcripts, expressed in the hippocampus of adult female rats, was approx. $1 / 3$ of that in the liver for $5 a$-reductase type 1, approx. $1 / 300$ of that in the male prostate for $5 a$-reductase type 2, approx. $1 / 10$ of that in the male prostate for AR, and approx. 1/100 of that in the adrenal for StAR (Table 1).

Mass-spectrometric determination of androgen and allopregnanolone levels in the female hippocampus

The concentration of T, DHT and Allo was determined for adult female and male rat hippocampus using a chromatogram analysis of the fragmented ions of steroid-derivatives. We chose the hippocampus at Proestrus stage for steroid determination since RT-PCR analysis revealed no estrous cycle-related fluctuation of the expression for $5 a$-reductase (types 1 and 2) which is required for synthesis of DHT and Allo. Results are summarized in Figures 2 and 3. T, DHT and Allo were derivatized with picolinoyl before application to LC-MS/MS to increase the accuracy of determination by improving the limit of quantification (LOQ) (Table S2).

In the chromatographic profiles of the fragmented ion of T-17picolinoyl-ester, DHT-17-picolinoyl-ester and Allo-3-picolinoyl-ester, a single clear peak was observed at $4.12,4.37$ and $8.43 \mathrm{~min}$, respectively (Figurea 2A1, 2B1 and 2C1). For these steroids, the retention time of the observed steroid peak was the same as that of standard steroid. To confirm the assay accuracy, the hippocampal homogenate spiked with known amounts of the steroids was prepared and its concentration of steroid was determined 296 (Table S3). The LOQs were defined in Table S2 as the lowest value with an acceptable accuracy (90-110\%) and precision (i.e. $\mathrm{RSD}<10 \%)$. The results of intra- and inter-assay were shown in Table S2. The RSD for intra- and inter-assay was less than $5.1 \%$ and $7.2 \%$, respectively.

Hippocampal level of androgens in female were $1.1 \mathrm{nM}$ for $\mathrm{T}$ and

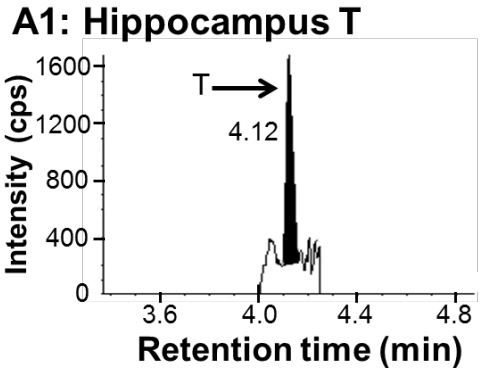

B1: Hippocampus DHT
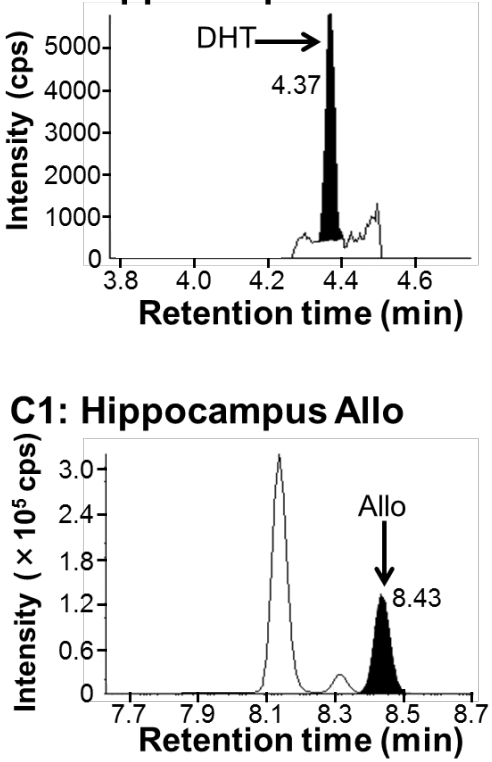
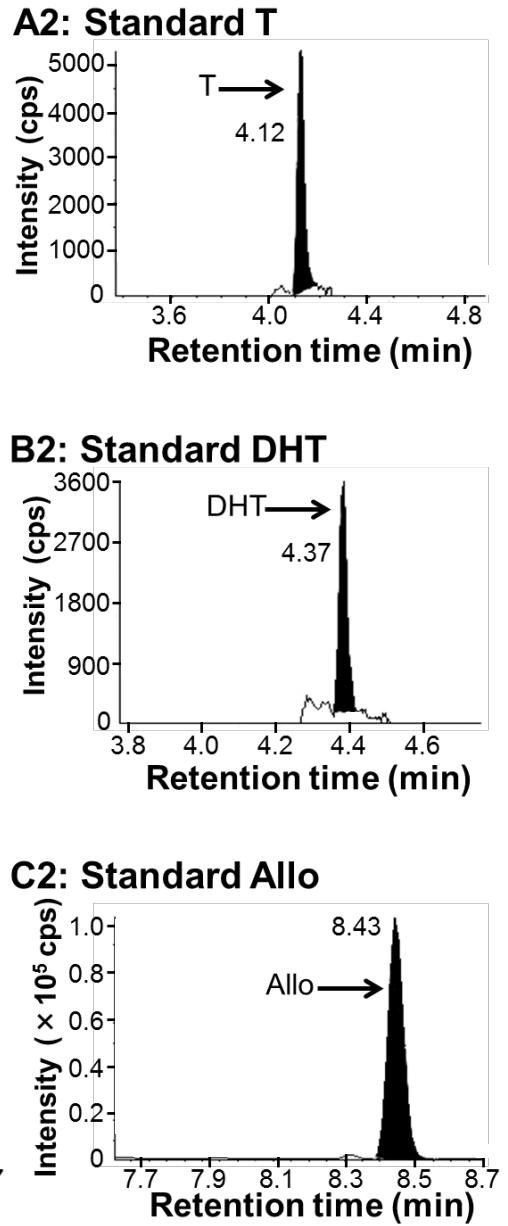

Figure 2: Mass-spectrometric analysis of hippocampal sex-steroids (12-week-old female rats), LC-MS/MS ion chromatograms of T (A), DHT(B) and Allo(C). (A1), (B1) and (C1) represent the chromatograms of the fragmented ions of each steroid from the hippocampus. Shaded portions indicate the intensity of fragmented ions of $T(m / z=253)$, DHT $(\mathrm{m} / \mathrm{z}=203)$ and Allo $(\mathrm{m} / \mathrm{z}=283.3)$, respectively. (A2), (B2) and (C2) represent the chromatograms of the fragmented ions of the standard steroids. The vertical axis indicates the intensity of the fragmented ion. The horizontal axis indicates the retention time of the fragmented ion, $\mathrm{t}=4.12 \mathrm{~min}$ for $\mathrm{T}, 4.37 \mathrm{~min}$ for $\mathrm{DHT}$, and $8.43 \mathrm{~min}$ for Allo, respectively. The time of sample injection to LC system was defined as $t=0 \mathrm{~min}$. Note that pre-purification step using normal phase HPLC before injection to LC system is very important to achieve high precision and good reproducibility of LC-MS/MS determination in order to avoid contamination of other steroids and fats. Steroid-derivatives or steroids were further separated with reversed phase LC-column before MS/MS. In the multiple reaction monitoring modes, the instrument monitored the $\mathrm{m} / \mathrm{z}$ transition (Table S2). 


\begin{tabular}{|l|l|l|}
\hline Enzyme or receptor & $\begin{array}{l}\text { Relative expression level in } \\
\text { female hippocampus }\end{array}$ & Reference organ \\
\hline 5a-reductase 1 & $1: 3$ & Liver \\
\hline 5a-reductase 2 & $1: 300$ & Prostate $^{c}$ \\
\hline AR & $1: 10$ & Prostate $^{\text {aR }}$ \\
\hline StAR & $1: 100$ & \\
\hline & adrenal \\
\hline
\end{tabular}

aValue of Proestrus sample was used because no fluctuation of mRNA level was observed between any stage of estrous cycle

${ }^{\mathrm{b}}$ Relative expression level in female hippocampus (Proestrus) is indicated with the ratio to that in reference organ

'Prostate was prepared from 12 week-old male rats

Table 1: Comparison of mRNA levels of steroidogenic enzymes or receptors between in hippocampus and in reference organ.

\begin{tabular}{|c|c|c|c|}
\hline \multicolumn{2}{|c|}{ Testosterone (T) } & \multicolumn{2}{c|}{ Dihydrotestosterone (DHT) } \\
\hline$(n M)^{\mathrm{b}}$ & $(\mathrm{ng} / \mathrm{g}$ wet weight) & $(\mathrm{nM})$ & (ng/g wet weight) \\
$1.10 \pm 0.28^{\mathrm{c}}\left(\mathrm{n}^{\mathrm{d}=12)}\right.$ & $0.32 \pm 0.08$ & $0.62 \pm 0.16(\mathrm{n}=7)$ & $0.18 \pm 0.05$ \\
\hline
\end{tabular}

(A) Hippocampal androgens (T and DHT) in female ${ }^{a}$

\begin{tabular}{|c|c|c|c|}
\hline \multicolumn{2}{|c|}{ Female (n=3) } & \multicolumn{2}{c|}{ Male $(\mathbf{n}=3)$} \\
\hline$(\mathrm{nM})$ & (ng/g wet weight) & $(\mathrm{nM})$ & $(\mathrm{ng} / \mathrm{g}$ wet weight) \\
$16.4 \pm 2.3$ & $5.2 \pm 0.7$ & $1.00 \pm 0.71$ & $0.32 \pm 0.23$ \\
\hline
\end{tabular}

(B) Hippocampal Allo in female and male

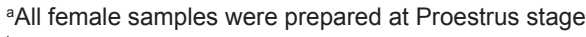

${ }^{\mathrm{b}}$ Concentration in $\mathrm{nM}$ is calculated using the average volume of $0.124 \mathrm{~mL}$ for one whole hippocampus that has $0.124 \pm 0.002 \mathrm{~g}$ wet weight $(n=44)$. We assumed that tissue having $1 \mathrm{~g}$ of wet weight has an approximate volume of

Table 2: Mass spectrometric analysis of the concentration of androgens ( $T$ and $\mathrm{DHT}$ ) or Allopregnanolone (Kerr JE et al.) in the hippocampus and plasma of adult female rats.

$0.62 \mathrm{nM}$ for DHT, respectively (Figure 3a). The level of hippocampal T was much higher than that of plasma $\mathrm{T}(\sim 0.1 \mathrm{nM})$ in female. In contrast to androgens, the levels of Allo in the female hippocampus was $16.4 \mathrm{nM}$ which was 16 times higher than that in male (Figure $3 \mathrm{~B} ; \mathrm{t}=6.52, \mathrm{df}=4$, $\mathrm{p}=0.0029$ )

In order to compare hippocampal levels of steroids with plasma steroids, we converted ng/g wet weight to $\mathrm{nM}$ concentration via the following estimation (Table 2). First, $1 \mathrm{~mL}$ of plasma (93\% is water) is assumed to have $1 \mathrm{~g}$ weight, as $1 \mathrm{~mL}$ of water has $1 \mathrm{~g}$ weight. Second, we assume that the hippocampal tissue having $1 \mathrm{~g}$ of wet weight has an approx. volume of $1 \mathrm{~mL}$, as nearly $78 \%$ of the brain tissue consists of water [29]. Consideration of specific volume of protein and lipids $(0.7-1.0 \mathrm{~mL} / \mathrm{g})$ in the brain further support this assumption [11,30,31]. After dividing by the individual hippocampal volume $(0.124 \pm 0.002$ wet weight for one whole hippocampus of 12 weeks old female rat, $\mathrm{n}=44$ ), the levels of T, DHT, Allo in the hippocampus were calculated. Based on these considerations, $0.18 \mathrm{ng} / \mathrm{g}$ wet weight of DHT in the hippocampus at Pro corresponds to $0.62 \mathrm{nM}$ (Table 2).

\section{Localization of steroidogenic enzymes and receptors in female hippocampus}

The localization and presence of P450 (17a), P450arom and StAR in the hippocampus of adult female rats were demonstrated by Immunohistochemical staining. P450 (17 $\alpha$ ), P450arom and StAR were mainly localized in pyramidal neurons in CA1-CA3 region as well as granule cells in dentate gyrus [9] (Figure 4A-4C). Immuno staining of glial cells for these enzymes was much weaker than that of principal neurons. In addition to steroidogenic enzymes, we also demonstrated the localization for AR and ERa in hippocampal slices of female. AR and ERa also localized in pyramidal and granule neurons (Figures $4 \mathrm{D}$ and $4 \mathrm{E}$ ). It should be noted that AR immuno reactivity was the most prominent at CA1pyramidal neurons, whereas region difference in staining pattern of ERa was not observed. The staining pattern of steroidogenic enzymes and receptors in female hippocampus was almost the same as that of male hippocampus $[2,4,11,12,32]$.

\section{Discussion}

\section{No significant sex difference in hippocampal steroidogenic systems (mRNA and protein)}

Surprisingly, the expression levels of sex steroidogenic enzymes including $5 a$-reductase (types 1 and 2 ) and StAR were not significantly different between female and male hippocampus (Figure 1). Furthermore, no cyclic fluctuation of expression levels of these enzymes was observed across the estrous cycle. Other steroidogenic enzymes which are required for androgen or estrogen synthesis including P450 (17 $\alpha$ ), P450 arom and 17 $\beta$-HSD (T synthase) also exhibited neither sex difference nor estrous cycle-dependent fluctuation [1], suggesting the constant catalytic activity for steroids in the hippocampus. It may be possible that the localization of enzymes or receptors is sex different even with the equal level of mRNA expression. Therefore, we investigated the localization of enzymes including P450 (17 $\alpha$ ) and P450arom or receptors including AR and ERa in female hippocampus with immunohistochemistry in current study, resulting in the same pattern as male (Figure 4) $[2,4,11,12]$. These results suggest that hippocampal synthesis activity of androgen or Allo is independent of sex. In fact, $5 a$-reductase activity was almost equal between in male and female from the data with high correlation between hippocampal $\mathrm{T}$ and hippocampal DHT (Figure 3C). All data of hippocampal $\mathrm{T}$ (x-axis) and DHT (y-axis) from both sexes were aligned in a straight line with high correlation (Figure $3 \mathrm{C}$ ), implying that DHT production was only dependent on substrate $\mathrm{T}$ concentration. Here we present analysis at only Proestrus stage, and further analysis at other estrus stages may help better understanding. In addition, we also measured corticosterone (CORT) level in the female hippocampus, and CORT was not considerably elevated upon sacrifice or anesthetics treatment. Only approx. $200 \mathrm{nM}$ CORT was penetrated to the hippocampus, although plasma CORT transiently elevated to approx. $1500 \mathrm{nM}$, which is similar to the situation in male rat hippocampus [33]. These results suggest that effects by sacrifice -stress on the hippocampus may not be serious.

\section{Sex difference in hippocampal androgen and allo levels}

The current study revealed that female hippocampus is able to synthesize androgens ( $\mathrm{T}$ and $\mathrm{DHT}$ ) in addition to estrogens. In female, hippocampal $\mathrm{T}(\sim 1 \mathrm{nM})$ is much higher (nearly 10 fold) than plasma $\mathrm{T}(\sim 0.1 \mathrm{nM})[1]$, implying that the contribution of plasma $\mathrm{T}$ to hippocampal $\mathrm{T}$ is very weak. This is very different from male in which $70-80 \%$ of hippocampal $\mathrm{T}$ is derived from plasma $\mathrm{T}$ [21]. Since female plasma DHT would be lower than plasma T $(\sim 0.1 \mathrm{nM})$, plasma DHT cannot contribute to the production of hippocampal DHT $(\sim 0.6 \mathrm{nM})$ in female. Majority of female hippocampal DHT should be therefore synthesized from hippocampal-synthesized T. Although male hippocampus synthesizes androgens and estrogens $[2,11,21,33,34]$, sex difference exists in the level of hippocampal steroids. The hippocampal level of androgens (T and DHT) is significantly high in male than in the female. The levels of $\mathrm{T}$ and DHT in the female hippocampus were 15 times lower than those in male (Figure 3A). In contrast to androgens, hippocampal Allo level was 16 times higher in female than in male. What generates such a sex difference in hippocampal steroid level? It may be due to the difference in precursor steroids supplied via blood circulation between female and male. Most abundant precursor 
A

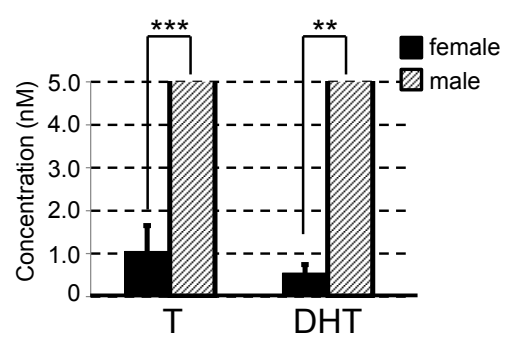

C

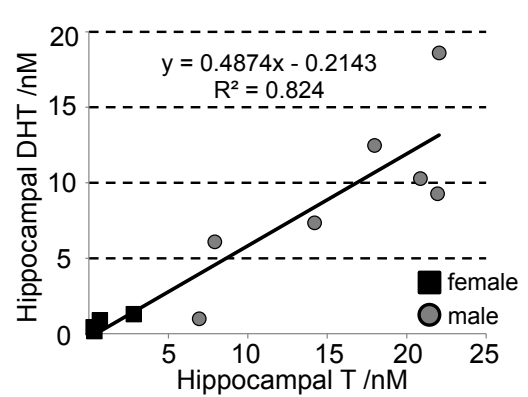

B

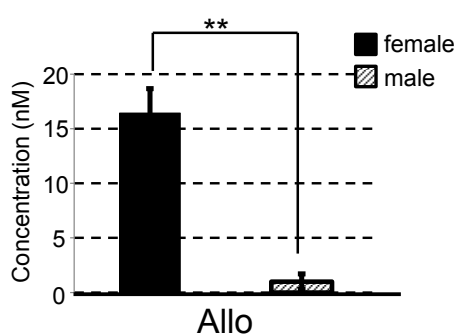

Figure 3: (A) Comparison of hippocampal androgens ( $T$ and DHT) level between male and female. Data are expressed as Mean \pm SEM. Hippocampal level of androgens was significantly different between female and male $(t=5.94, d f=7, p=0.00057 \mathrm{for} T$, and $t=4.12, d f=6, p=0.0062$ for DHT, respectively). (B) Comparison of hippocampal Allo level between male and female. Data are expressed as Mean \pm SEM. Hippocampal level of Allo was significantly different between female and male $(t=6.52, d f=4, p=0.0029)$. (C) Relationship between hippocampal T and DHT for individual rats. Male data are taken from (Hojo Y et al., 2009). Number of animals is 7 for male and 4 for female, respectively. Statistical significance, ${ }^{* *} p<0.01,{ }^{* * *} p<0.001$

\section{A: P450(17a)}

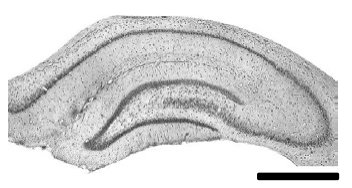

D:AR

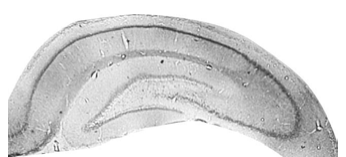

B: P450arom

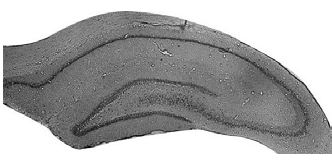

\section{C: StAR}

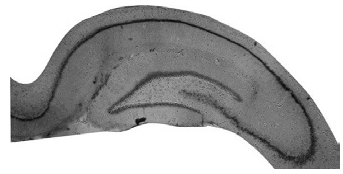

E: ERa

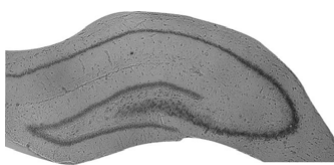

Figure 4: Immunohistochemical staining for P450(17a) (A), P450arom (B) StAR (C), AR (D) and ERa (E) in the hippocampus of 12 week-old female rat at Proestrus stage. The coronal section of whole hippocampus $(20 \mathrm{~mm}$ thickness) was used. Scale bar, $800 \mathrm{~mm}$.

steroids for synthesis of sex steroids in the hippocampus may be PROG (precursor of Allo) originally produced by ovary or adrenal gland (20$50 \mathrm{nM}$ in plasma) in female, and T (precursor of DHT) from testis ( 15 $\mathrm{nM})$ in male, respectively $[21,1]$. Almost all hippocampal androgens ( $\mathrm{T}$ and DHT) in female were hippocampus-synthesized from PROG, because the level of plasma $\mathrm{T}(\sim 0.1 \mathrm{nM})$ in female was much lower than that of hippocampal T [1].

\section{Earlier studies on female androgens in the brain}

The concentration of $\mathrm{T}$ and DHT in adult female hippocampus had not yet been accurately determined in previous studies. Although one study reported the presence of androgens with RIA method [6], the numerical concentrations were not written due to very low levels. On the other hand, a significant concentration of Allo has been demonstrated using RIA [6,14], GC-MS [15,16] and LC-MS/MS [17]. The concentration of Allo was approximately $6 \mathrm{ng} / \mathrm{g}(\sim 20 \mathrm{nM})$ in female hippocampus at Proestrus $[6,14]$. On the other hand Allo was very low approximately $0.4 \mathrm{ng} / \mathrm{g}(\sim 1 \mathrm{nM})$ in male adult whole brain $[15,16]$ or undetectable in male cultured hippocampus. These concentrations are qualitatively similar to those observed in the current study. Compared to male, physiological role of androgens in female hippocampus is not clear. One of the possible physiological roles might be suppression of depression, since supplementation of androgens in female decreased 
Citation: Hojo Y, Okamoto M, Kato A, Higo S, Sakai F, et al. (2014) Neurosteroid Synthesis in Adult Female Rat Hippocampus, Including Androgens and Allopregnanolone. J Steroids Hormon Sci S4: 002. doi:10.4172/2157-7536.S4-002

a depression-like behavior of aged mice [7]. Extensive studies on physiological roles of female androgen should be needed.

\section{Acknowledgements}

Dr. S. Homma (Aska Pharmamedical) is acknowledged for steroid analysis.

\section{References}

1. Kato A, Hojo Y, Higo S, Komatsuzaki Y, Murakami G, et al. (2013) Female hippocampal estrogens have a significant correlation with cyclic fluctuation of hippocampal spines. Front Neural Circuits 7: 149

2. Hojo Y, Hattori TA, Enami T, Furukawa A, Suzuki K, et al. (2004) Adult male rat hippocampus synthesizes estradiol from pregnenolone by cytochromes P45017alpha and P450 aromatase localized in neurons. Proc Natl Acad Sci U S A 101: 865-870.

3. Hojo Y, Murakami G, Mukai H, Higo S, Hatanaka Y, et al. (2008) Estrogen synthesis in the brain--role in synaptic plasticity and memory. Mol Cell Endocrinol 290: 31-43.

4. Kawato S, Hojo Y, Kimoto T (2002) Histological and metabolism analysis of P450 expression in the brain. Methods Enzymol 357: 241-249.

5. Okamoto M, Hojo Y, Inoue K, Matsui T, Kawato S, et al. (2012) Mild exercise increases dihydrotestosterone in hippocampus providing evidence for androgenic mediation of neurogenesis. Proc Natl Acad Sci U S A 109: 13100 13105.

6. Frye CA, Bayon LE (1999) Mating stimuli influence endogenous variations in the neurosteroids 3alpha,5alpha-THP and 3alpha-Diol. J Neuroendocrinol 11 839-847.

7. Frye CA, Walf AA (2009) Depression-like behavior of aged male and female mice is ameliorated with administration of testosterone or its metabolites. Physiol Behav 97: 266-269.

8. Matsui D, Sakari M, Sato T, Murayama A, Takada I, et al. (2002) Transcriptional regulation of the mouse steroid 5alpha-reductase type II gene by progesterone in brain. Nucleic Acids Res 30: 1387-1393.

9. Pardridge WM, Mietus LJ (1979) Transport of steroid hormones through the rat blood-brain barrier. Primary role of albumin-bound hormone. J Clin Invest 64: $145-154$.

10. Higo S, Hojo Y, Ishii H, Komatsuzaki Y, Ooishi Y, et al. (2011) Endogenous synthesis of corticosteroids in the hippocampus. PLoS One 6: e21631.

11. Kimoto T, Tsurugizawa T, Ohta Y, Makino J, Tamura H, et al. (2001) Neurosteroid synthesis by cytochrome p450-containing systems localized in the rat brain hippocampal neurons: N-methyl-D-aspartate and calciumdependent synthesis. Endocrinology 142: 3578-3589.

12. Mukai H, Tsurugizawa T, Murakami G, Kominami S, Ishii H, et al. (2007) Rapid modulation of long-term depression and spinogenesis via synaptic estrogen receptors in hippocampal principal neurons. J Neurochem 100: 950-967.

13. Kerr JE, Allore RJ, Beck SG, Handa RJ (1995) Distribution and hormona regulation of androgen receptor $(A R)$ and $A R$ messenger ribonucleic acid in the rat hippocampus. Endocrinology 136: 3213-3221.

14. Frye CA, Petralia SM, Rhodes ME (2000) Estrous cycle and sex differences in performance on anxiety tasks coincide with increases in hippocampa progesterone and 3alpha,5alpha-THP. Pharmacol Biochem Behav 67: 587596.

15. Ebner MJ, Corol DI, Havlíková H, Honour JW, Fry JP (2006) Identification of neuroactive steroids and their precursors and metabolites in adult male rat brain. Endocrinology 147: 179-190.

16. Meffre D, Pianos A, Liere P, Eychenne B, Cambourg A, et al. (2007) Steroid profiling in brain and plasma of male and pseudopregnant female rats after traumatic brain injury: analysis by gas chromatography/mass spectrometry. Endocrinology 148: 2505-2517.

17. Ishihara Y, Kawami T, Ishida A, Yamazaki T (2013) Allopregnanolonemediated protective effects of progesterone on tributyltin-induced neuronal injury in rat hippocampal slices. J Steroid Biochem Mol Biol 135: 1-6.

18. Frye CA, Walf AA (2002) Changes in progesterone metabolites in the hippocampus can modulate open field and forced swim test behavior of proestrous rats. Horm Behav 41: 306-315.

19. Frye CA, Walf AA (2004) Hippocampal 3alpha,5alpha-THP may alter depressive behavior of pregnant and lactating rats. Pharmacol Biochem Behav 78: 531-540

20. Smith SS, Shen H, Gong QH, Zhou X (2007) Neurosteroid regulation of GABA(A) receptors: Focus on the alpha4 and delta subunits. Pharmacol Ther 116: 58-76.

21. Hojo $\mathrm{Y}$, Higo S, Ishii H, Ooishi $\mathrm{Y}$, Mukai H, et al. (2009) Comparison between hippocampus-synthesized and circulation-derived sex steroids in the hippocampus. Endocrinology 150: 5106-5112.

22. Fusani L, Gahr M (2006) Hormonal influence on song structure and organization: the role of estrogen. Neuroscience 138: 939-946.

23. Kimoto T, Ishii H, Higo S, Hojo Y, Kawato S (2010) Semicomprehensive analysis of the postnatal age-related changes in the mRNA expression of sex steroidogenic enzymes and sex steroid receptors in the male rat hippocampus. Endocrinology 151: 5795-5806.

24. Gorski RA, Mennin SP, Kubo K (1975) The neural and hormonal bases of the reproductive cycle of the rat. Adv Exp Med Biol 54: 115-153.

25. Caruso D, Pesaresi M, Maschi O, Giatti S, Garcia-Segura LM, et al. (2010) Effect of short-and long-term gonadectomy on neuroactive steroid levels in the central and peripheral nervous system of male and female rats. $J$ Neuroendocrinol 22: 1137-1147.

26. Shinzawa K, Ishibashi S, Murakoshi M, Watanabe K, Kominami S, et al. (1988) Relationship between zonal distribution of microsomal cytochrome P-450s (P 450(17) alpha,lyase and P-450C21) and steroidogenic activities in guinea-pig adrenal cortex. J Endocrinol 119: 191-200.

27. Jakab RL, Horvath TL, Leranth C, Harada N, Naftolin F (1993) Aromatase immunoreactivity in the rat brain: gonadectomy-sensitive hypothalamic neurons and an unresponsive "limbic ring" of the lateral septum-bed nucleusamygdala complex. J Steroid Biochem Mol Biol 44: 481-498.

28. Clark BJ, Wells J, King SR, Stocco DM (1994) The purification, cloning, and expression of a novel luteinizing hormone-induced mitochondrial protein in MA-10 mouse Leydig tumor cells. Characterization of the steroidogenic acute regulatory protein (StAR). J Biol Chem 269: 28314-28322.

29. Mcllwain $\mathrm{H} \mathrm{BH}$ (1985) Biochemistry and the Central Nervous System. Edinburgh: Churchill Livingstone.

30. Xie XS, Stone DK (1986) Isolation and reconstitution of the clathrin-coated vesicle proton translocating complex. J Biol Chem 261: 2492-2495.

31. Tanford C, Nozaki Y, Reynolds JA, Makino S (1974) Molecular characterization of proteins in detergent solutions. Biochemistry 13: 2369-2376.

32. Tabori NE, Stewart LS, Znamensky V, Romeo RD, Alves SE, et al. (2005) Ultrastructural evidence that androgen receptors are located at extranuclear sites in the rat hippocampal formation. Neuroscience 130: 151-163.

33. Hojo Y, Higo S, Kawato S, Hatanaka Y, Ooishi Y, et al. (2011) Hippocampa synthesis of sex steroids and corticosteroids: essential for modulation of synaptic plasticity. Front Endocrinol (Lausanne) 2: 43.

34. Kawato S (2004) Endocrine disrupters as disrupters of brain function: a neurosteroid viewpoint. Environ Sci 11: 1-14.
This article was originally published in a special issue, Neurosteroids handled by Editor. Dr. Salman Azhar, Stanford University, USA 